\title{
Oestrogens promote tumorigenesis in a mouse model for colitis-associated cancer
}

\author{
Jarom Heijmans, ${ }^{1}$ Mattheus C B Wielenga, ${ }^{1}$ Sanne Liesbeth Rosekrans, ${ }^{1}$ \\ Jooske F van Lidth de Jeude, ${ }^{1}$ Joris Roelofs, ${ }^{2}$ Patrick Groothuis, ${ }^{3}$ Antwan Ederveen, ${ }^{3}$ \\ Eveline S M de Jonge-Muller, ${ }^{4}$ Izak Biemond, ${ }^{4}$ James C H Hardwick, ${ }^{4}$ Geert D'Haens, ${ }^{1}$ \\ Daniel W Hommes, ${ }^{4,5}$ Vanesa Muncan, ${ }^{1}$ Gijs $R$ van den Brink ${ }^{1}$
}

\section{- Additional material is published online only. To view please visit the journal online (http://dx.doi.org/10.1136/ gutjnl-2012-304216) \\ ${ }^{1}$ Tytgat Institute for Liver \& Intestinal Research and Department of \\ Gastroenterology and Hepatology, Academic Medical Center, Amsterdam, The Netherlands \\ ${ }^{2}$ Department of Pathology, Academic Medical Center, Amsterdam, The Netherlands ${ }^{3}$ Merck, Sharpe and Dohme, Women's Health Department, Oss, The Netherlands ${ }^{4}$ Department of \\ Gastroenterology and Hepatology, Leiden University Medical Center, Leiden, The Netherlands \\ ${ }^{5}$ Center for Inflammatory Bowel Diseases, University of California Los Angeles, Los Angeles, USA}

\section{Correspondence to} Jarom Heijmans, Tytgat Institute for Liver \& Intestina Research, Academic Medical Center, Meibergdreef 69-71, 1105 BK Amsterdam,

The Netherlands; j.heijmans@amc.nl, Gijs R van den Brink, Tytgat Institute for Liver \& Intestinal Research, Academic Medical Center, Meibergdreef 6971,1105 BK Amsterdam, The Netherlands; g.r.vandenbrink@amc.nl

$\mathrm{JH}$ and MCBW contributed equally

Received 26 November 2012 Revised 5 January 2013 Accepted 8 January 2013 Published Online First 13 February 2013

To cite: Heijmans J, Wielenga MCB, Rosekrans SL, et al. Gut 2014;63:310-316.

\section{ABSTRACT}

Background Hormone replacement therapy increases the risk of developing ulcerative colitis in postmenopausal women. Chronic intestinal inflammation predisposes to colon cancer development, but effects of female hormones on colitis-associated cancer development have not been examined.

Aim To investigate the role of female hormones in the dextran sodium sulfate (DSS)-azoxymethane (AOM) mouse model for colitis-associated cancer.

Design We performed ovariectomies, or sham operations, on mice, and supplemented these animals with indicated hormones. Additionally, we used oestrogen receptor $\alpha$ or $\beta$ (Er $\alpha$ or Er $\beta$ ) mutant mice. To study colitis or colitis-associated cancer, we used DSS only, or DSS and AOM, respectively.

Results Ovariectomy protects female mice against colitis-associated tumour development. Hormone replacement in ovariectomised mice with either oestradiol (E2), medroxyprogesterone acetate or a combination of both suggests that oestrogens are the ovary-derived factor that promotes tumour development in the context of inflammatory damage. E2-treated animals showed increased clinical symptoms and II-6 production upon DSS-induced colitis and enhanced epithelial proliferation. Treatment with E2 markedly increased the numbers of polyps in ovariectomised mice and also strongly promoted tumour progression with all E2-treated animals developing at least one invasive adenocarcinoma, whereas, placebo-treated animals developed adenomas only. Using Er mutant mice, we find that the protumorigenic effect of oestrogen depends on both Er $\alpha$ and $\operatorname{Er} \beta$.

Conclusions Our results suggest that oestrogens promote inflammation-associated cancer development by impairing the mucosal response to inflammatory damage.

\section{INTRODUCTION}

Patients with inflammatory bowel disease (IBD) are at an increased risk of developing colorectal cancer (CRC). The risk of developing CRC correlates with the duration, extent and severity of inflammation. ${ }^{12}$ Factors driving inflammation-associated cancers are different from sporadically occurring cancers as they seem to be driven by a distinct sequence of DNA mutations. ${ }^{3}$

One of the important risk factors for the development of sporadic adenomas and CRC is male

\section{Significance of this study}

What is already known about this subject?

- Female hormones increase the risk for developing inflammatory bowel disease.

- Female hormones protect against sporadic colorectal cancer development.

- Oestrogens have known pro- and antitumorigenic effects.

What are the new findings?

- Endogenous female hormones deteriorate colitis-associated cancer development in vivo.

- Of female hormones, oestrogens are responsible for proinflammatory and protumorigenic effects.

- The effect of oestrogens on development of colitis-associated cancer depends on both oestrogen receptors $\alpha$ and $\beta$.

How might it impact on clinical practice in the foreseeable future?

- Our data suggest that care should be taken with prescribing hormone replacement therapy in women with inflammatory bowel disease.

gender. Women have a delayed development of adenomas and colon cancers. ${ }^{45}$ This gender disparity may depend on a protective effect of female hormones in premenopausal women. The protective effect of female hormones was suggested by the outcome of the Women's Health Initiative. In these large randomised placebo-controlled trials, the combination of oestrogen and medroxyprogesterone acetate (MPA) reduced the number of colon cancers by $37 \%$ compared with placebo at 5 years follow-up. ${ }^{67}$ Treatment with oestrogens alone did not significantly affect the risk of colon cancer development. ${ }^{8}{ }^{9}$

Recently, it was shown in a large cohort study, that the risk of developing IBD is increased in postmenopausal women who were/are past/current users of female hormones (either oestrogens or oestrogens in combination with progestins). ${ }^{11}{ }^{11}$ However, the role of female hormones in colitis-associated cancer development has not been examined. We therefore set out to investigate a role for female hormones in an experimental model for colitis-associated cancer 
development. In line with the data that suggest a predisposing role for oestrogens in the development of ulcerative colitis, we find that oestrogens promote tumour incidence and progression in the context of intestinal inflammation.

\section{MATERIAL AND METHODS}

\section{Animal experiments}

All experiments were performed according to the guidelines of the Experimental Animal Center of the Leiden University Medical Center (PDC), or the Animal Research Institute of the Academic Medical Center. $E r \alpha^{12}$ and $E r \beta^{13}$ knockout mice were ordered at Jacksons Laboratories and bred heterozygously to generate $\mathrm{Er \alpha}^{-/-}$or $\mathrm{Er}^{-/-}$animals. For all experiments, wildtype littermate control animals were used. All mouse experiments were performed on $\mathrm{C} 57 \mathrm{BL} / 6 \mathrm{~J}$ background.

Ovariectomy (OVX) was performed on all mice, unless indicated otherwise. For OVX, a sagittal cut of $1 \mathrm{~cm}$ was made on the back of mice. The skin was then manipulated over the fat pad where the ovarium resides. A small cut was made in the fat pad, and the ovarium was found and removed by cauterisation. The fat pad was closed by a single suture and the skin was closed using staples. Where indicated, a pellet containing either steroid hormone or placebo (Innovative Research of America) was implanted subcutaneously in the neck. One week after surgery, animals were enrolled in the dextran sodium sulfate (DSS)-azoxymethane (AOM) protocol, or the DSS protocol.

For the DSS-AOM protocol, on day 1, mice were injected with azoxymethane $(10 \mathrm{mg} / \mathrm{kg} /$ day; Sigma-Aldrich, Zwijndrecht, Netherlands), and drinking water was changed for water supplemented with $1.5 \%$ DSS weight per volume. On day 4, DSS was refreshed, and on day 6, DSS was changed back for regular drinking water. A similar cycle of DSS was repeated in week 4 and week 7. At 12 weeks of age, animals were sacrificed and colons were fixed in phosphate buffered saline (PBS) containing $4 \%$ formaldehyde. For the experiment with DSS only, animals received drinking water supplemented with 2\% DSS for 7 days. On day 8 animals, were sacrificed. One hour prior to sacrifice, all animals were injected with $200 \mu \mathrm{l} \mathrm{BrdU}(10 \mathrm{mg} / \mathrm{ml}$ in PBS; Sigma-Aldrich). Since the severity of DSS-AOM-induced tumorigenesis tends to vary between different experiments, we included placebo-treated animals in each experiment.

\section{Hormone replacement}

Slow-release pellets with hormones (Innovative Research of America) were based on an average weight of $25 \mathrm{~g} /$ mouse. Pellets contained the following steroid doses: oestradiol (E2): $0.025 \mathrm{mg}$ per 90-day slow-release pellet: $10 \mu \mathrm{g} / \mathrm{kg} /$ day. MPA: $7.5 \mathrm{mg}$ per 90-day slow-release pellet: $3.3 \mathrm{mg} / \mathrm{kg} /$ day. Placebo pellets were of similar size, but contained no steroids.

\section{Polyp counting}

After overnight fixation of colons, tissue was rinsed with PBS twice and immersed in 70\% ethanol overnight. Subsequently, polyps were counted. Polyp size was estimated using a ruler guide.

\section{DSS scoring system}

A standardised scoring system was used to assess the severity of colitis both clinically and histopathologically. ${ }^{14}$

\section{Tissue processing and immunohistochemistry}

Immunohistochemistry was performed as previously described. ${ }^{15}$ In short, tissue was fixed in 10\% ice-cold formalin embedded in paraffin. Sections of $4 \mu \mathrm{m}$ were deparaffinised in xylene and rehydrated. For immunohistochemistry, endogenous peroxidase was blocked using $0.3 \% \mathrm{H}_{2} \mathrm{O}_{2}$ in methanol. The sections were cooked in $0.01 \mathrm{M}$ citrate buffer $\mathrm{pH} 6.0$ for $20 \mathrm{~min}$ and incubated with the primary antibody in PBS with $1 \%$ bovine serum albumin and $0.1 \%$ Triton X-100. Antibody binding was visualised with Powervision horseradish peroxidase-labelled secondary antibodies, and diaminobenzidine for substrate development. All sections were counterstained with Mayer's haematoxylin. The following antibodies were used: mouse monoclonal anti-BrdU (clone BMC9318, Roche), rabbit monoclonal anticleaved-caspase 3 (\#9664, Cell signalling technologies).

\section{Cytokine bead analysis}

Intestines were lysed in nine volumes of Greenberger lysis buffer using a motorised Turrax homogeniser. The lysis was made out of a $2 \times$ buffer containing $150 \mathrm{mM} \mathrm{NaCl}, 15 \mathrm{mM}$ Tris, $1 \mathrm{mM} \mathrm{MgCl} 2,1 \mathrm{mM} \mathrm{CaCl} 2$ and $1 \%$ Triton X-100 $\mathrm{pH} 7.4$ prior to use, the $2 \times$ buffer was diluted $1: 1$ with $0.9 \% \mathrm{NaCl}$, and to $50 \mathrm{ml}$ buffer 1 tablet protease inhibitor (Roche) was added. Tissues were homogenised on ice, and after homogenisation they were put to rest on ice for $30 \mathrm{~min}$. Subsequently, the lysates were centrifuged for $7 \mathrm{~min}$ at 4000 rounds per minute (RPM) at $4^{\circ} \mathrm{C}$ in a microcentrifuge. The supernatant was put into a clean new tube and centrifuged for $10 \mathrm{~min}$ at 14000 $\mathrm{RPM}$ at $4^{\circ} \mathrm{C}$, and stored at $20^{\circ} \mathrm{C}$ for further analysis. Cytokine bead analysis (CBA) was performed on a mouse Th1 cytokine kit $(R \& D)$ according to the manufacturer's protocol.

\section{Statistical analysis}

All data are presented as mean \pm SE of the mean. For animal experiments, a Student $t$ test or a 1-way analysis of variance (ANOVA) test was used. For the analysis of the distribution of polyp size, 2-way ANOVA tests were used. All ANOVA tests were followed by Bonferroni's post-test for multiple comparisons. For survival analysis, Gehan-Breslow-Wilcoxon values were noted for comparison, corrected using Bonferroni's post-test for multiple comparisons, where $\mathrm{p}<0.05 / \mathrm{K}$ was regarded as significant. $\mathrm{K}$ was defined as the total number of analyses made.

\section{RESULTS}

Female hormones promote tumorigenesis in a mouse model for colitis-associated cancer

To analyse the role of female hormones in intestinal tumorigenesis in the context of inflammation, we used the AOM-DSS model. ${ }^{16}$ In this model, an injection with the carcinogen AOM is followed by three subsequent cycles of DSS to induce colitis (figure 1A). DSS causes direct damage to the colonic epithelium, eliciting a wound-healing response that markedly enhances AOM-induced tumorigenesis. We first examined whether female hormones influence colitis-associated cancer development. In female mice, endogenous production of female sex hormones can be largely abrogated by OVX. We therefore subjected female mice that had undergone OVX, or a sham operation in which the ovaries where left in situ, to the AOM-DSS model. During the cycles of DSS-induced colitis, we noticed that animals in which ovaries had been removed were slightly but not significantly protected from DSS-induced weight loss (data not shown). Surprisingly, we found that females who had undergone OVX were markedly protected from tumour development compared with sham operated mice (figure $1 \mathrm{~B}, 7.0$ vs 12.0 tumours per colon, $\mathrm{p}<0.001)$. Size distribution of the polyps did not differ between mice that had undergone OVX or a sham procedure (figure $1 \mathrm{C}$ ). These data suggest that female hormones promote tumorigenesis in the context of inflammation. 
Figure 1 Endogenous female hormones promote colitis-associated tumorigenesis. (A) Protocol for induction of colitis-associated tumours by azoxymethane combined with dextran sodium sulfate (AOM-DSS). Mice are injected with $10 \mathrm{mg} / \mathrm{kg}$ AOM on day 1 , and colitis is induced by addition of indicated amounts of DSS to the drinking water in weeks 1,4 and 7 . Animals are sacrificed for polyp count 12 weeks after the injection of AOM. (B) Polyp number of mice that had undergone ovariectomy (OVX), or sham operations, prior to AOM-DSS. (C) Distribution of polyp size after OVX or a sham procedure ${ }^{* *}=\mathrm{p}<0.01$.
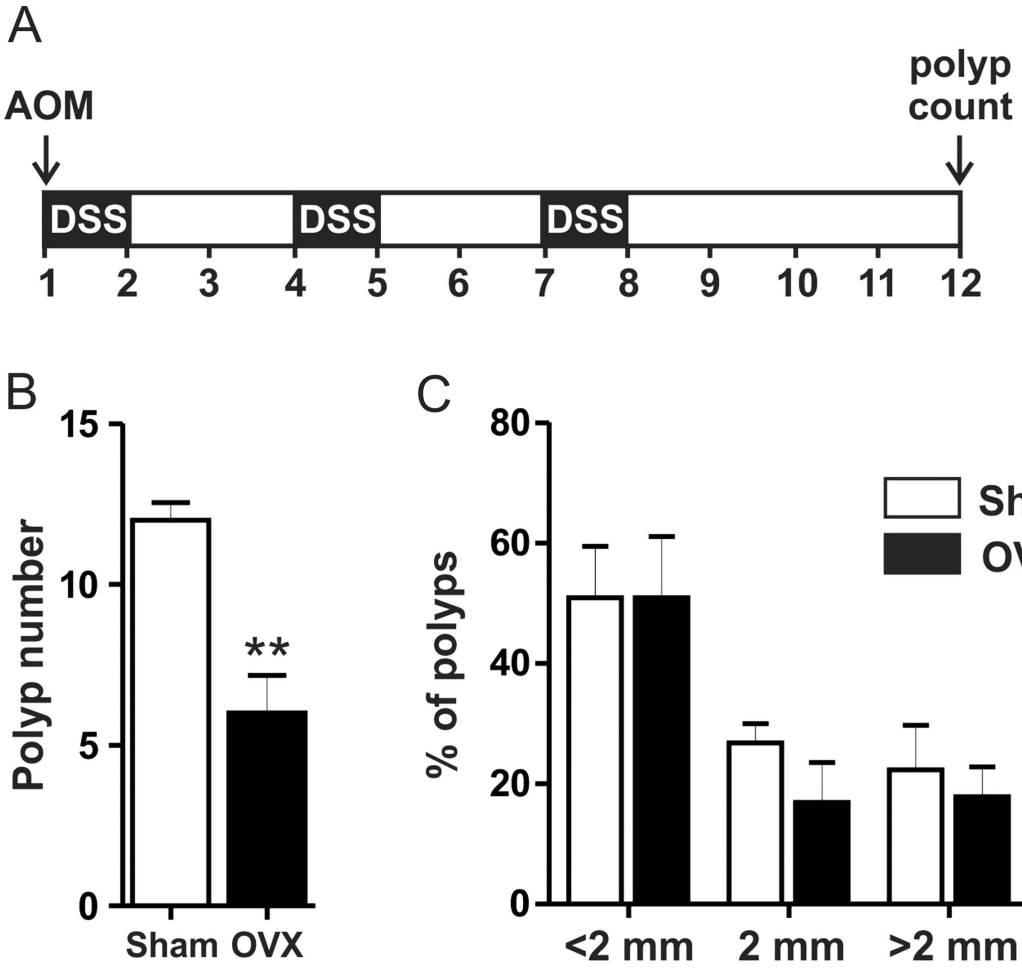

\section{Colitis-associated tumorigenesis is promoted by oestrogens}

We next set out to investigate which ovarian hormone is responsible for the promotion of colitis-associated cancer development. Therefore, we induced tumours in mice that underwent OVX, and in which we replaced endogenous hormones with slow-release pellets implanted subcutaneously. Animals were treated with pellets containing the oestrogen oestradiol (E2, $10 \mu \mathrm{g} / \mathrm{kg} /$ day), the progestin (MPA, $3.3 \mathrm{mg} / \mathrm{kg} /$ day) or with a combination of both. MPA is a synthetic progestagen that signals through the progesterone receptor, and is widely used as a contraceptive agent. Additionally, MPA in combination with oestrogen, was shown to be protective in sporadic colon cancer development in humans. ${ }^{6}$ The concentrations used were based on the IC50 required for inhibition of ovulation in rats, and allosterically converted to mice. ${ }^{17}$ To control for placement of the pellet, a placebo pellet was implanted in mice that did not undergo hormone replacement. OVX was performed together with the placement of the pellets 1 week prior to enrolment in the AOM-DSS protocol. Animals that received E2, either as monotherapy or in combination with MPA suffered more DSS-induced weight loss than control animals or animals that had received MPA monotherapy (figure $2 \mathrm{~A}, \mathrm{p}<0.01$ on day 8 for E2 vs placebo, $\mathrm{p}<0.001$ on day 8 for $\mathrm{E} 2+\mathrm{MPA}$ vs placebo). This resulted in reduced survival of mice that had received E2 $(p<0.01)$, but not for animals that had received the combination of E2 and MPA (figure 2B). We treated all groups with $1.5 \%$ DSS in the drinking water for a period of 5 days in weeks 1,4 and 7, since this was the highest level of DSS that was tolerated in E2-treated mice. Due to the low levels of DSS we gave to all animals, control mice showed minimal clinical symptoms (eg, weight loss, diarrhoea and rectal bleeding). Additionally, control animals developed only few tumours. Mice that received E2 showed a dramatic 10-fold increase in polyp development compared with placebo-treated mice (figure 2C,F, 1.5 vs 16 polyps/mouse in placebo and E2-treated mice, respectively, $\mathrm{p}<0.001)$. In agreement with the survival data, animals that had received MPA together with E2 were partially protected from
E2-induced tumorigenesis (figure 2C, 5.6 polyps/mouse in MPA + E2-treated mice, $\mathrm{p}<0.001)$. E2 promoted tumour incidence and also tumour progression, as we observed a shift towards larger polyp size in E2-treated mice (figure 2D, $\mathrm{p}<0.01$ ). To examine how E2 affects mucosal homeostasis, we assessed numbers of proliferating and apoptotic cells in the normal appearing mucosa at the moment of sacrifice. We found that E2 significantly increased the number of Ki-67 positive cells, whereas the number of apoptotic (active caspase- 3 positive) cells was very low and unchanged by treatment with E2 (figure 2E). The polyps were assessed by an expert GI pathologist (JR) on one longitudinal microscopic section of the colon of every animal. It was found that all animals treated with E2 developed adenocarcinomas with invasive growth (figure $2 \mathrm{G}$ ), whereas no adenocarcinomas were observed in any of the animals that had received placebo ( $\mathrm{p}<0.01$ Fisher's exact test).

To examine if the tumour-promoting effect of oestrogen was specific to female mice, we also enrolled male mice that had undergone a sham operation in the AOM-DSS model. Similar to females, E2 treatment caused enhanced weight loss and decreased survival in DSS-AOM-treated males (see online supplementary figure S1A,B). Additionally, an increase in tumour number was seen in males receiving E2 compared with placebo (see online supplementary figure S1C), albeit to a lesser extent than in females $(3.6 \pm 2.7$ vs $7.6 \pm 3.6, p<0.05)$. Likewise, a trend towards larger polyps was observed, although not significant (see online supplementary figure S1D). Thus, oestrogens promote both tumour incidence and growth in the context of inflammation.

Oestrogens aggravate clinical symptoms of disease, but not histological severity of colitis

E2-treated mice showed increased weight loss during the cycles of DSS in the AOM-DSS model. At the time of sacrifice however, the weight of different treatment groups had reverted to the levels that had been measured before the start of DSS-AOM. Weight loss is a sensitive measure of the severity of 

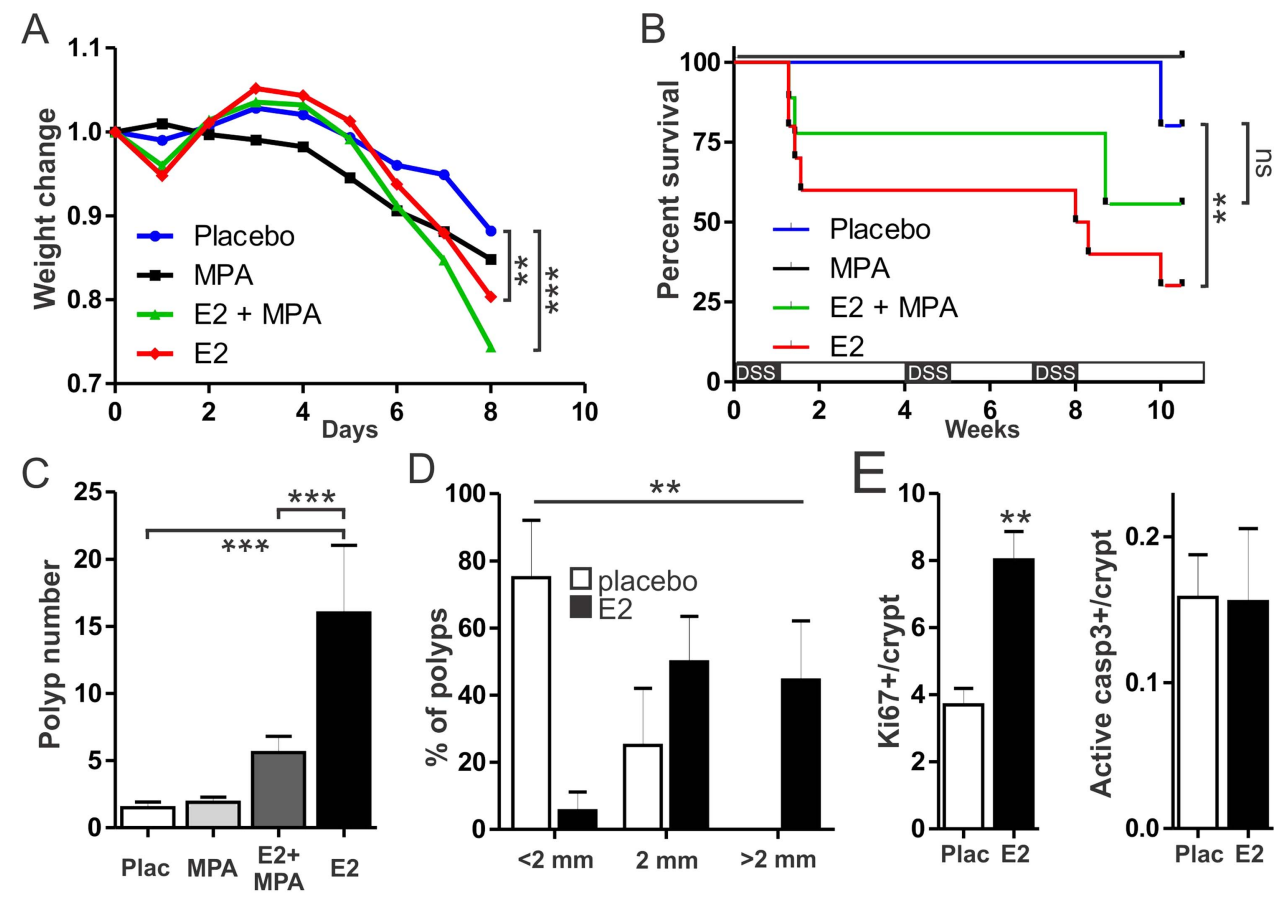

F
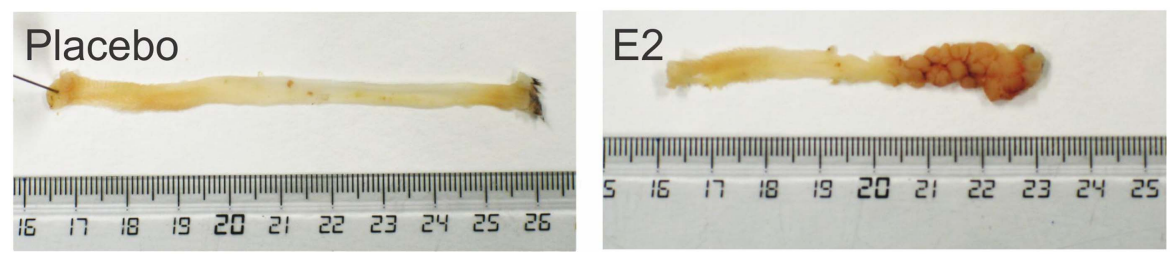

G
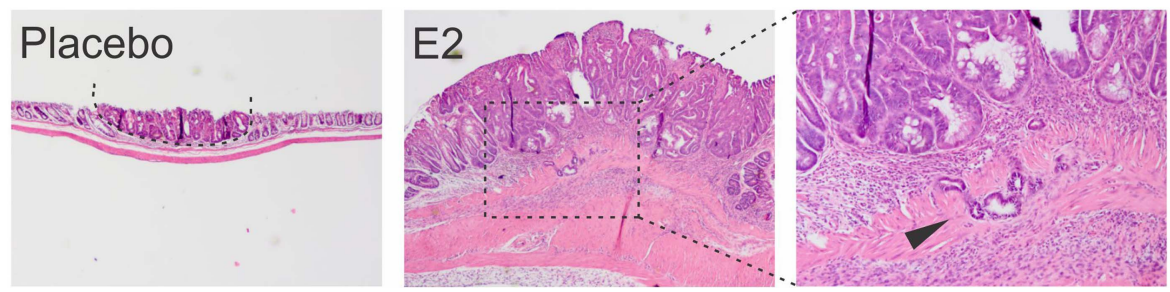

Figure 2 Oestrogens promote colitis-associated cancer development. Female mice that underwent ovariectomy (OVX), were implanted with hormone or placebo pellets as indicated, and were subjected to azoxymethane (AOM)-dextran sodium sulfate (DSS). (A) Mice on E2 alone or E2 +medroxyprogesterone acetate (MPA) combination therapy lost significantly more weight than mice implanted with placebo pellets. (B) Mice on E2 replacement therapy after OVX showed significantly decreased survival compared with controls. (C) Polyp counts show that E2 replacement promotes tumour development in OVX mice in the AOM-DSS model, whereas MPA significantly protects against the tumour-promoting effects of E2. (D) E2 replacement results in a significant shift towards larger polyps in the AOM-DSS model. (E) E2 replacement increases the number of BrdU positive cells, but not caspase-3 positive cells. (F) Representative pictures of colons of a mouse from the placebo group versus a mouse on E2 replacement therapy. (G) Representative photomicrographs show H\&E section of a small polyp in an animal from the placebo group, and an invasive adenocarcinoma (arrow head indicates area of invasive growth) in an animal treated with E2 ${ }^{* *}=\mathrm{p}<0.01,{ }^{* *}=\mathrm{p}<0.001$.

colitis in the DSS model, which in turn is linked to tumorigenesis. Thus, increased severity of colitis could explain the tumour-promoting properties seen in animals treated with E2. Although previous reports have shown colitogenic effects of E2, these experiments were performed without OVX, in the presence of endogenous hormone production. ${ }^{18}$ We therefore repeated these experiments and examined if E2 may aggravate DSS-induced colitis. As in the previous experiment, we subjected mice to OVX and injected these animals with E2 or placebo pellets. They subsequently received a high dose of DSS $(2.5 \%)$ in their drinking water for 7 days.

E2 aggravated colitis development as judged by clinical symptoms: weight loss, diarrhoea and rectal blood loss (figure $3 \mathrm{~A}, \mathrm{~B}$, see online supplementary figure S2A). However we observed no significant differences in the histopathological score of colitis that was scored by an expert gastrointestinal pathologist (JR), blinded to the treatment of the mice (see online supplementary figure S2B). As in the AOM-DSS experiment, E2 replacement increased the rate of proliferation in DSS-treated animals, but left the number of apoptotic epithelial cells unchanged (figure 3C). We performed a Th1 cytokine bead array to examine the influence of oestrogens on a panel of inflammatory cytokines (Ifn $\gamma$, Tnf $\alpha$, Il-12 p40, Il-6, Il-10, Mcp-1). Ifn $\gamma$ was below the level of detection. Of the other cytokines, only Il-6 was significantly increased by oestrogen replacement compared with animals implanted with placebo pellets (figure $3 \mathrm{D}$, 
Figure 3 Oestrogens increase clinical disease, but not the histological severity of colitis. Female mice that underwent ovariectomy, were implanted with pellets containing E2 or placebo, and were subsequently subjected to DSS-induced colitis. (A) Disease activity index, a score that includes weight loss, diarrhoea and rectal blood loss was increased by E2. (B) Animals receiving E2 suffered significantly more weight loss than placebo-treated animals.

(C) Immunohistochemistry shows that E2 significantly increased the number of BrdU positive cells in $S$ phase in E2-treated animals, but did not affect the low number of apoptotic cells marked by active caspase-3. (D) E2 promoted the secretion of II- 6 in DSS-induced colitis * $=p<0.05$, ${ }^{* *}=\mathrm{p}<0.01$.

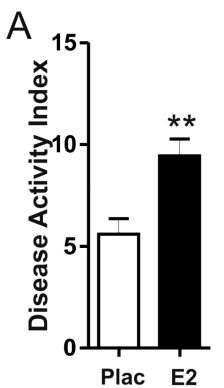

B
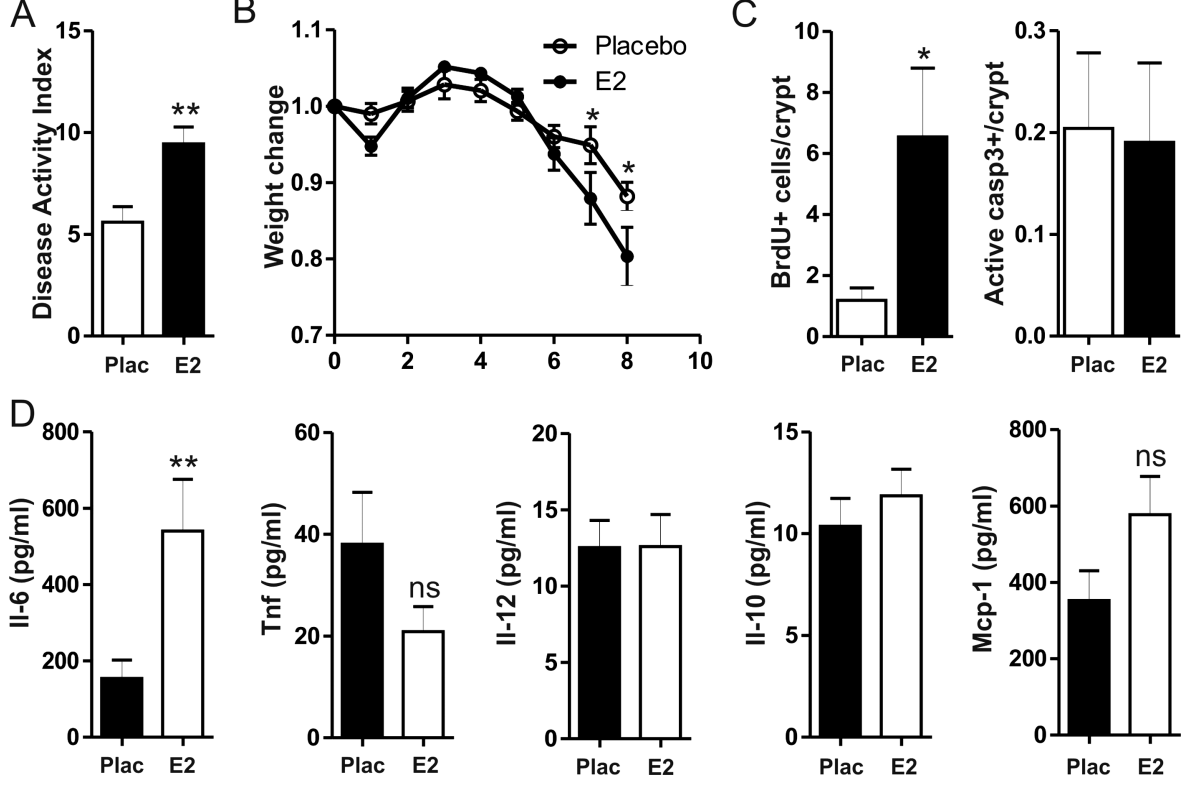

$\mathrm{p}<0.01)$. These results suggest that during DSS-induced inflammation, oestrogens increase clinical severity of inflammation, which correlates with increased mucosal concentrations of Il-6 and increased epithelial proliferation. However, the gross histological severity of colitis remains unaltered. These observations where made in two independent experiments with identical results.

\section{Oestrogen signalling promotes tumorigenesis through both Ero and $\operatorname{Er} \boldsymbol{\beta}$}

E2 signals through two distinct oestrogen receptors (Ero and $\operatorname{Er} \beta)$. When E2 binds the receptors, they form homo- or heterodimers, and translocate from the cytosol to the nucleus where they act as transcription factors. The oestrogen receptors, Er $\alpha$ and $\operatorname{Er} \beta$, are products of different genes localised on different chromosomes, and with distinct expression patterns. They can mediate distinct effects of oestrogens and have pleiotropic effects on cancer development. ${ }^{19}$

To examine which oestrogen receptor is responsible for the oncogenic effect of E2 in colitis-associated cancer development, we bred $\mathrm{Era}^{+/-}$and $\mathrm{Er}^{+/-}$mice and compared homozygous mutant mice with their littermate wild-type mice in the AOM-DSS model. Similar to our previous experiments, all animals were subjected to OVX prior to the start of AOM-DSS, and mice similarly received slow-release pellets with either E2 or with placebo.

During the DSS cycles in the protocol, wild-type control mice treated with E2 exhibited marked weight loss compared with placebo-treated wild-type control mice as expected $(\mathrm{p}<0.0001$ in littermate control animals of both $\mathrm{ErO}^{-/-}$and $\mathrm{Er}^{-/-}$mice). $\mathrm{Er \alpha}^{-/-}$mice were completely protected against the effect of E2 on DSS-induced weight loss (figure 4A). By contrast, weight loss in $\mathrm{Er}^{-/-}$mice was even slightly, though not significantly, higher than in wild-type control mice (figure 4B). Both $E r \alpha^{-/-}$and $\mathrm{Er}^{-/-}$mice were protected against the enhanced proliferation observed in the epithelium of E2-treated wild-type mice (figure 4C). When analysing polyp numbers, we found that E2 consistently and reproducibly increased the tumour load throughout our experiments. Despite their distinct effects on the clinical severity of colitis during the DSS cycles, both $E r \alpha^{-/-}$ and $\mathrm{Er}^{-/-}$mice were protected from E2-induced tumorigenesis (figure 4D,G). Additionally, E2 consistently and significantly increased tumour size in wild-type littermate control animals, but not in $\mathrm{Er}^{-/-}$and $\mathrm{Er} \beta^{-/-}$mice (figure 4E,F and H,I). Taken together, these results suggest that the tumour-promoting effects of E2 require both Er $\alpha$ and $\operatorname{Er} \beta$. However, there may be differences in the way in which these receptors modulate tumorigenesis. Based on the DSS-induced weight loss, we find that Ero promotes tumorgenesis by aggravating inflammation, whereas, $\operatorname{Er} \beta$ has inflammation-independent effects on tumours in the context of intestinal inflammation.

\section{DISCUSSION}

The large prospective randomised trials of the Women's Health Initiative have firmly established that hormone replacement therapy with a combination of oestrogen and MPA protects against the development of CRC. ${ }^{6-8}$ Recently, large epidemiological studies have suggested that oestrogens promote the development of ulcerative colitis. ${ }^{10} 11$ We find that ovariectomised mice are protected against the development of colitis-associated cancer development. Using different combinations of replacement therapy, we find that oestrogens promote colitis-associated cancer development. Additionally, oestrogens promote clinical signs of colitis, such as weight loss and the severity of diarrhoea, but had no effects on histological scores as judged by H\&E staining. The increased clinical symptoms correlated with elevated Il-6 production, but levels of Tnf $\alpha$, Il-12 and Il-10 were not affected by oestrogens. A colitogenic effect of oestrogens in the DSS model has previously been reported by others in mice that had not undergone OVX. ${ }^{18}$ In these experiments, it was shown that oestrogens aggravate DSS-induced colitis both clinically and histologically. In our experiments, we find that oestrogens increase clinical symptoms but not the histology of colitis. Potentially, this discrepancy depends on the fact that we used an approximate 80 -fold lower dose of oestrogens in our experiments. Although we find no alterations in the histological colitis score or in separate features of intestinal histology, animals suffer from increased clinical disease and show increased secretion of Il-6. This may suggest that similar to the study of Verdú et al, oestrogen increases the inflammatory milieu upon DSS-induced colitis, which may contribute to carcinogenesis. Interestingly, in the same study by Verdú et al, oestrogens had a protective effect 

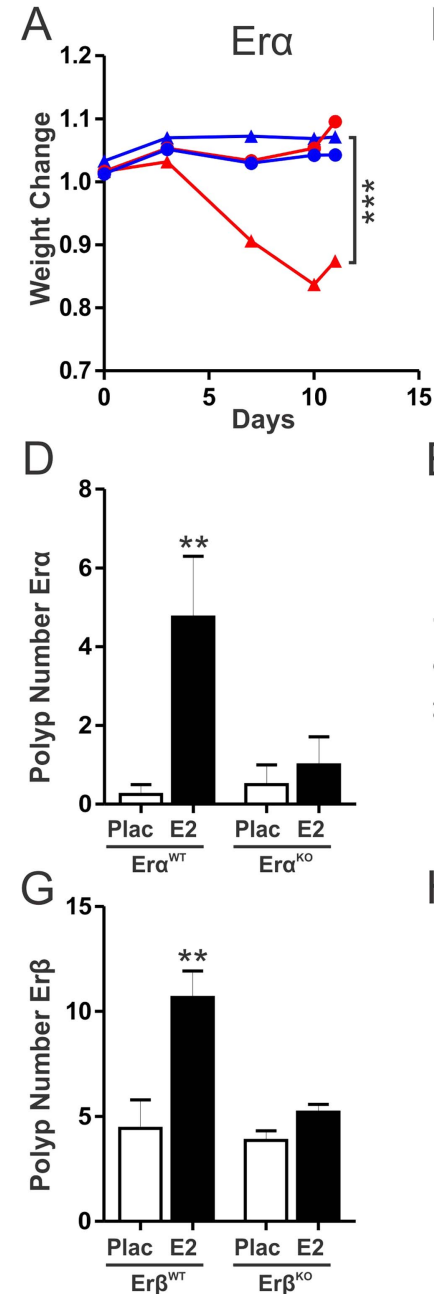

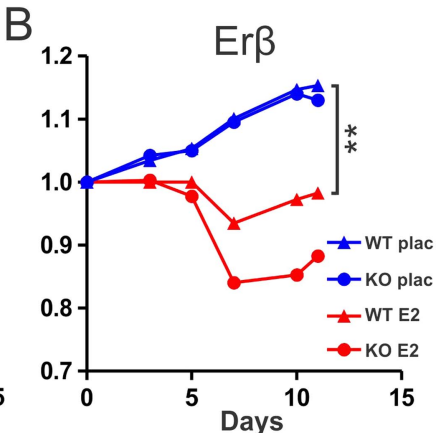

E
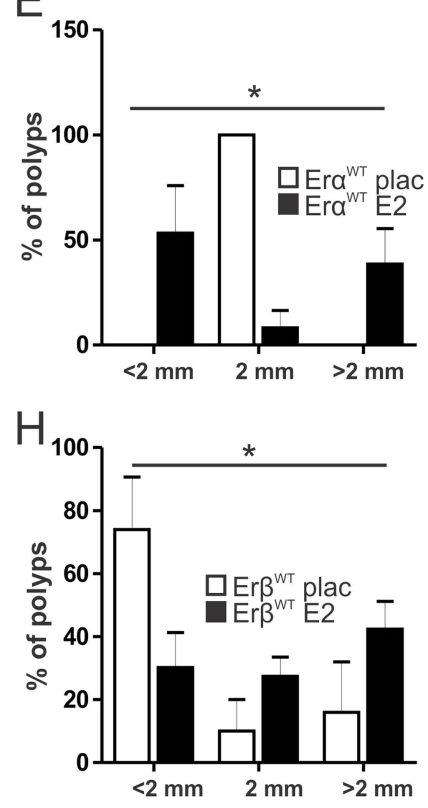

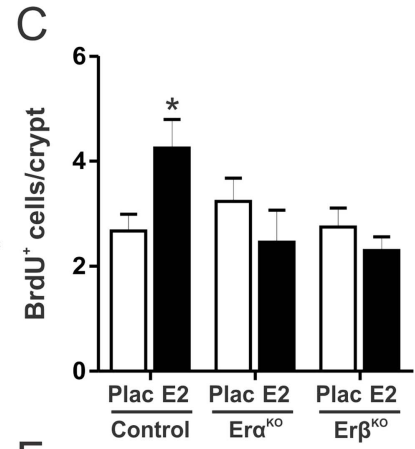

F
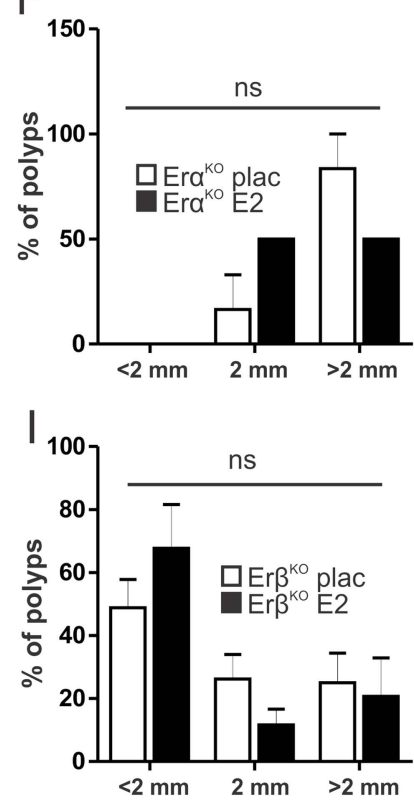

Figure 4 Oestrogens promote colitis-associated tumorigenesis through both Er $\alpha$ and $\operatorname{Er} \beta$. Female $\operatorname{Er} \alpha$ and $\operatorname{Er} \beta$ mutant and their wild-type littermate control animals underwent ovariectomy (OVX), and were subjected to azoxymethane-dextran sodium sulfate after replacement with E2 or a placebo pellet. (A) Weight curve shows that Ero mutant mice were completely protected against the weight loss-promoting effect of E2. (B) In Er $\beta$ mutant mice, there was a trend towards increased weight loss in E2-treated animals compared with placebo. (C) Counting of BrdU-positive cells shows that absence of both Er $\alpha$ and $E r \beta$ protected against the enhanced epithelial proliferation observed in E2-treated mice. (D) Polyp numbers of wild-type and Er $\alpha$ mutant mice treated with E2 or placebo after OVX. (E) In wild-type animals, E2 replacement significantly promoted tumour progression. (F) By contrast, in Er $\alpha$ mutant littermates, the distribution of polyp size was similar between E2 and placebo-treated mice. (G) Polyp numbers of wild-type and $\operatorname{Er} \beta$ mutant mice treated with E2 or placebo after OVX. (H) In wild-type animals, E2 replacement significantly promoted tumour progression. (I) By contrast, in Er $\beta$ mutant littermates, the distribution of polyp size was similar between E2 and placebo-treated mice ${ }^{*}=p<0.05,{ }^{* *}=p<0.01,{ }^{* * *}=p<0.001$.

on colitis induced with the hapten dinitrobenzene. This is an intriguing finding in the light that oestrogens may increase the risk of ulcerative colitis but not Crohn's disease in humans. Thus, the effects of oestrogens may depend on the nature of the inflammatory response in both mice and humans. As judged by the different roles of Er $\alpha$ and $\operatorname{Er} \beta$ in DSS-induced weight loss, it seems that the two oestrogen receptors may have opposing effects on the severity of colitis. This could be related to the differential expressions of the two receptors between the epithelium and lamina propria cells, a question we did not pursue in this study. This, however, is an observation that warrants further research into the role of the oestrogen receptors in the development of colitis.

Tumour induction with DSS-AOM may vary between experiments due to environmental factors or batch to batch variation of reagents used. Therefore, we used placebo-treated animals in addition to oestrogen-treated animals in each experiment. We find that despite their divergent effects on the severity of clinical symptoms during colitis, both $\operatorname{Er} \alpha$ and $\operatorname{Er} \beta$ are required for the tumour-promoting effects of oestrogens. By contrast with littermate control animals, we find that animals lacking either Er $\alpha$ or $\operatorname{Er} \beta$ become insensitive to the tumour-promoting effects of oestrogens. Interestingly, $\mathrm{Er}^{-/-}$mice have increased tumour size compared with control animals in the absence of oestrogens (placebo-treated ovariectomised mice). This may reflect an oestrogen-independent function of Er $\alpha$, since these animals lack both endogenous and exogenous oestrogens. Our studies contrast with a recent report that suggests that $\operatorname{Er} \beta$ mutant mice have increased tumour development in the AOM-DSS model of colitis-associated cancer development. ${ }^{20}$ In this study, it was found that $\operatorname{Er} \beta$ mutant mice have a more rapid tumour development compared with wild-type littermates in the AOM-DSS model. The $\operatorname{Er} \beta$ mutants had an increased number of tumours at week 9, whereas tumour load was equal to wild-type mice at week 16. The most noticeable difference between the two experimental approaches is the fact that we performed OVX in 
all our studies, thereby abrogating endogenous sex hormone production, whereas in the study by Saleiro et al, the ovaries were left in situ. OVX plus oestrogen replacement is often performed in $\mathrm{Er}$ mutant mice as the lack of one of the receptors may modulate oestrogen levels and affect signalling through the remaining receptor. A well known example is the increased plasma levels of oestrogens that are present in Ero mutant mice. ${ }^{21}$ A similar effect may exist in $\operatorname{Er} \beta$ mutant mice, as $\operatorname{Er} \beta$ plays an important role in the ovaries where it is expressed in the granulosa cells and regulates granulosa cell differentiation. ${ }^{22}$ $\operatorname{Er} \beta$ mutant mice display aberrant regulation of oestrogen production by the ovaries ${ }^{22}$ which may influence outcomes in animal models. These effects can be circumvented by performing OVX plus hormone replacement.

In the AOM-DSS model, tumorigenesis depends on DSS-induced tissue damage and the subsequent epithelial repair. It has recently been demonstrated that efficient repair of inflammatory mucosal injury is important to prevent ongoing inflammation and epithelial proliferation. ${ }^{23}$ This was demonstrated in Egfr mutant mice which show an impaired epithelial response to a proinflammatory environment on an $\mathrm{Il}-10$ mutant background resulting in an exaggerated response to DSS with overexpression of proinflammatory cytokines, excess weight loss and increased mortality. The mice showed a particular overexpression of Il-6, a cytokine that plays a key role in colitis-associated cancer development. $^{2425}$ This excess production of Il-6 likely plays a key role in the ongoing proliferative response in both Egfr mutant and E2-treated mice. Finally, the Egfr mutant mice develop more tumours on an $\mathrm{Il}-10$ mutant background and show enhanced tumour progression with development of invasive adenocarcinomas. Thus, E2 may promote colitis-associated colon cancer development by impairing the resolution of inflammatory injury similar to Egfr mutant mice.

Increased production of Il-6 in E2-treated mice may be the result of impaired mucosal repair, but may also be more direct. It was previously shown that oestrogens promote the production of Il-6 in peritoneal macrophages in vitro and in vivo. ${ }^{26}$ Similar to our own results in these macrophages, oestrogens had no effect on Il-12 production and only little effect on Tnfo. ${ }^{26}$ On the other hand, the effect of oestrogens on Il- 6 production in the context of colitis is in contrast with studies showing that oestrogens inhibit Il-6-dependent bone resorption, ${ }^{27}$ and can suppress Il-6-dependent tumorigenesis in the liver. ${ }^{28}$ This suggests that effects of oestrogens on Il-6 production are highly context-dependent.

In conclusion, we find that oestrogens increase severity of colitis and colitis-associated cancer development. Both Er $\alpha$ and $\operatorname{Er} \beta$ are required for the tumour-promoting role of oestrogens. The tumour-promoting properties of oestrogens correlate with increased production of Il-6 and an exaggerated proliferative response of the colonic epithelium which may indicate that E2 compromises mucosal repair after inflammatory injury. Since colitis-associated cancer development is a relatively rare occurrence, there is no clinical information on the effect of female hormone replacement in the development of colitis-associated cancer in humans. Our results seem to suggest, however, that care should be taken to assure that women with ulcerative colitis who are on hormone replacement therapy, undergo adequate colonoscopy screening for colon cancer development.

Contributors JH, MCBW, SR, JFLJ, ESMJ-M, IB and VM performed experiments. $J H, M C B W, V M$ and $J R$ analysed data. PG, AE, JCHH, GD'H and DWH discussed data and commented on results and on the manuscript. JH, MCBW, VM and GRB wrote the manuscript. GRB supervised all experiments.
Funding This research was funded by an unrestricted grant of MSD, The Netherlands, and grant UL-2010-4667 from the Dutch Cancer Society.

Competing interests None.

Provenance and peer review Not commissioned; externally peer reviewed.

\section{REFERENCES}

1 Ekbom A, Helmick C, Zack M, et al. Ulcerative colitis and colorectal cancer. A population-based study. N Engl J Med 1990;323:1228-33.

2 Ekbom A, Helmick C, Zack M, et al. Increased risk of large-bowel cancer in Crohn's disease with colonic involvement. Lancet 1990;336:357-9.

3 Ullman TA, Itzkowitz SH. Intestinal inflammation and cancer. Gastroenterology 2011;140:1807-16

4 Regula J, Rupinski M, Kraszewska E, et al. Colonoscopy in colorectal-cancer screening for detection of advanced neoplasia. N Engl J Med 2006;355:1863-72.

5 Ferlitsch M, Reinhart K, Pramhas $\mathrm{S}$, et al. Sex-specific prevalence of adenomas, advanced adenomas, and colorectal cancer in individuals undergoing screening colonoscopy. JAMA 2011;306:1352-8.

6 Chlebowski RT, Wactawski-Wende J, Ritenbaugh C, et al. Estrogen plus progestin and colorectal cancer in postmenopausal women. N Engl J Med 2004;350:991-1004.

7 Rossouw JE, Anderson GL, Prentice RL, et al. Risks and benefits of estrogen plus progestin in healthy postmenopausal women: principal results From the Women's Health Initiative randomized controlled trial. JAMA 2002;288:321-33.

8 Anderson $\mathrm{GL}$, Limacher $\mathrm{M}$, Assaf $\mathrm{AR}$, et al. Effects of conjugated equine estrogen in postmenopausal women with hysterectomy: the Women's Health Initiative randomized controlled trial. JAMA 2004;291:1701-12

9 Ritenbaugh C, Stanford JL, Wu L, et al. Conjugated equine estrogens and colorectal cancer incidence and survival: the Women's Health Initiative randomized clinical trial. Cancer Epidemiol Biomarkers Prev 2008;17:2609-18.

10 Khalili H, Higuchi LM, Ananthakrishnan AN, et al. Oral contraceptives, reproductive factors and risk of inflammatory bowel disease. Gut 2013;62:1153-9.

11 Khalili H, Higuchi LM, Ananthakrishnan AN, et al. Hormone Therapy Increases Risk of Ulcerative Colitis but not Crohn's Disease. Gastroenterology 2012;143:1199-206.

12 Lubahn DB, Moyer JS, Golding TS, et al. Alteration of reproductive function but not prenatal sexual development after insertional disruption of the mouse estrogen receptor gene. Proc Natl Acad Sci USA 1993;90:11162-6.

13 Krege JH, Hodgin JB, Couse JF, et al. Generation and reproductive phenotypes of mice lacking estrogen receptor beta. Proc Natl Acad Sci U S A 1998;95:15677-82.

14 Cooper HS, Murthy SN, Shah RS, et al. Clinicopathologic study of dextran sulfate sodium experimental murine colitis. Lab Invest 1993;69:238-49.

15 Heijmans J, Muncan V, Jacobs RJ, et al. Intestinal tumorigenesis is not affected by progesterone signaling in rodent models. PLoS One 2011;6:e22620.

16 Neufert C, Becker C, Neurath MF. An inducible mouse model of colon carcinogenesis for the analysis of sporadic and inflammation-driven tumor progression. Nat Protoc 2007;2:1998-2004.

17 Reagan-Shaw S, Nihal M, Ahmad N. Dose translation from animal to human studies revisited. FASEB J 2008;22:659-61.

18 Verdu EF, Deng Y, Bercik P, et al. Modulatory effects of estrogen in two murine models of experimental colitis. Am J Physiol Gastrointest Liver Physiol 2002;283: G27-36.

19 Thomas C, Gustafsson JA. The different roles of ER subtypes in cancer biology and therapy. Nat Rev Cancer 2011;11:597-608.

20 Saleiro D, Murillo G, Benya RV, et al. Estrogen receptor-beta protects against colitis-associated neoplasia in mice. Int J Cancer 2012.

21 Couse JF, Korach KS. Estrogen receptor null mice: what have we learned and where will they lead us? Endocr Rev 1999;20:358-417.

22 Couse JF, Yates MM, Deroo BJ, et al. Estrogen receptor-beta is critical to granulosa cell differentiation and the ovulatory response to gonadotropins. Endocrinology 2005; 146:3247-62.

23 Dube $P E$, Yan F, Punit $S$, et al. Epidermal growth factor receptor inhibits colitis-associated cancer in mice. J Clin Invest 2012;122:2780-92.

24 Bollrath J, Phesse TJ, von Burstin VA, et al. gp130-mediated Stat3 activation in enterocytes regulates cell survival and cell-cycle progression during colitis-associated tumorigenesis. Cancer Cell 2009;15:91-102.

25 Grivennikov S, Karin E, Terzic J, et al. IL-6 and Stat3 are required for survival of intestinal epithelial cells and development of colitis-associated cancer. Cancer Cell 2009:15:103-13

26 Calippe B, Douin-Echinard V, Delpy L, et al. 17Beta-estradiol promotes TLR4-triggered proinflammatory mediator production through direct estrogen receptor alpha signaling in macrophages in vivo. J Immunol 2010;185:1169-76.

27 Jilka RL, Hangoc G, Girasole G, et al. Increased osteoclast development after estrogen loss: mediation by interleukin-6. Science 1992;257:88-91.

28 Naugler WE, Sakurai T, Kim S, et al. Gender disparity in liver cancer due to sex differences in MyD88-dependent IL-6 production. Science 2007;317:121-4 


\section{GUT}

\section{Oestrogens promote tumorigenesis in a mouse model for colitis-associated cancer}

Jarom Heijmans, Mattheus C B Wielenga, Sanne Liesbeth Rosekrans, et al.

Gut 2014 63: 310-316 originally published online February 13, 2013

doi: 10.1136/gutjnl-2012-304216

Updated information and services can be found at:

http://gut.bmj.com/content/63/2/310.full.html

These include:

Data Supplement "Supplementary Data"

http://gut.bmj.com/content/suppl/2013/02/11/gutjnl-2012-304216.DC1.html

References This article cites 27 articles, 11 of which can be accessed free at: http://gut.bmj.com/content/63/2/310.full.html\#ref-list-1

Email alerting service

Receive free email alerts when new articles cite this article. Sign up in the box at the top right corner of the online article.

Topic Articles on similar topics can be found in the following collections Collections

Notes

To request permissions go to:

http://group.bmj.com/group/rights-licensing/permissions

To order reprints go to:

http://journals.bmj.com/cgi/reprintform

To subscribe to BMJ go to:

http://group.bmj.com/subscribe/ 